\title{
Phase behavior of selected artificial lipids
}

\author{
Andreas Zumbuehl ${ }^{\mathrm{a}, \mathrm{b}, *}$, Bodo Dobner ${ }^{\mathrm{c}}$, Gerald Brezesinski ${ }^{\mathrm{d}}$ \\ a Department of Chemistry, University of Fribourg, Chemin du Musée 9, 1700 Fribourg, Switzerland \\ ${ }^{\mathrm{b}}$ Swiss National Centre of Competence in Research in Chemical Biology, Switzerland \\ ${ }^{\mathrm{c}}$ Institute of Pharmacy, Martin Luther University of Halle-Wittenberg, Wolfgang-Langenbeck-Str. 4, 06120 Halle (Saale), Germany \\ ${ }^{\mathrm{d}}$ Max Planck Institute of Colloids and Interfaces, Science Park Potsdam-Golm, Am Muehlenberg 1, 14476 Potsdam, Germany
}

\begin{abstract}
The flexibility of biomembranes is based on the physical-chemical properties of their main components glycerophospholipids. The structure of these modular amphiphilic molecules can be modified through organic synthesis making it possible to study specific physical-chemical effects in detail. In particular, the roles of the hydrophobic tails of the phospholipids and their hydrophobic/hydrophilic interfacial backbone on the phase behaviour are highlighted. The spatial orientation of the glycerol backbone changes from sn-1,2 to sn- 1,3 phospholipids leading to an increase of the in-p bne area of the molecu $\mathrm{k}$. The larger distance between the hydrophobic tails can lead to membrane leaflet interdigitation. The introduction of methyl side groups in the hydrophobic tails increases the fluidity of the bilayer. Depending on the position of the methyl branches partial interdigitation is observed. In the case of bolaamphiphiles, methyl side groups have a similar effect on the fluidity, but interdigitation cannot occur.
\end{abstract}

\section{Introduction}

The function of a biological membrane is to act as an enclosing and separating unit for the various ingredients and organelles of a cell [1]. However, besides this compartment formation the biological membrane is responsible for cell-cell-interactions and signal transduction [2]. The membrane has to ensure the lateral diffusion and lateral organization of non-lipid components like proteins [3] and to enable them to reach their active conformation [4]. This requires a biological membrane to maintain a fluid-like character and at the same time to organize in a more ordered environment around proteins (lipid rafts) [5]. This flexibility of the membrane is based on physical-chemical properties resulting from the amphiphilic character of its main components: glycerophospholipids.

Glycerophospholipids exist in a large variety of organized structures especially when hydrated. At a certain temperature, the structure of a polymorphic form depends not only on the chemical structure of the lipid molecule but also on variables such as hydration degree, ionic strength and $\mathrm{pH}$. X-ray diffraction techniques (Small- and Wide-angle $\mathrm{X}$-ray scattering (SAXS/WAXS)) are mainly used to determine the phase structure. DSC (differential scanning calorimetry) is used to determine the temperature and thermodynamical parameters of phase transitions occurring during heating or cooling the sample. Under physiological conditions $\left(37^{\circ} \mathrm{C}\right.$ and $\left.\mathrm{pH} 7.4\right)$ most of the membrane lipids form lamellar gel or liquid-crystalline phases, but non-lamellar liquid-crystalline phases such as hexagonal or cubic ones have also been observed. Most studies

\footnotetext{
* Corresponding author.

E-mail address: andreas.zumbuehl@unifr.ch (A. Zumbuehl).
}

focus on the transition from a gel phase with highly ordered alltrans-fatty acid chains (or even a subgel phase with ordering of entire molecules) into a liquid-crystalline phase with molten chains (gauche conformers) characterized by a diffuse halo in the WAXS pattern. Gel to liquid-crystalline phase transitions can be induced by changes in temperature (thermotropic behavior), hydration (lyotropic behavior), pressure, as well as by changing the ionic strength or $\mathrm{pH}$ of the aqueous phase.

In general, the rich polymorphism of glycerophospholipids is an intriguing property, not observed to the same extent in other classes of chemical compounds. The functional role of different structures remains far from being completely understood, especially in such a complex out-of-equilibrium system like the biological membrane. Chemical modifications of phospholipids therefore allow the study of specific structure/property relationships and to substantially better understand lipid polymorphism and its biological significance. In this review, we therefore concentrate on the thermotropic phase behavior of selected chemically modified lipids with modifications in the hydrophobic and hydrophilic parts as well as in the region connecting these parts of an amphiphilic molecule.

\section{Glycerophospholipids - modular molecules}

Glycerophospholipids can be subdivided into 7 modular units (see Fig. 1). Central to the molecule is its $C_{3}$-glycerol backbone. From here, typically, two hydrophobic tails and a hydrophilic head group are branched off, each connected via a chemical linker. The standard linker for the fatty chains is an ester function, for the head group, a phosphate diester is common. The chains are categorized according to their length and saturation. A hexadecyl chain as depicted 


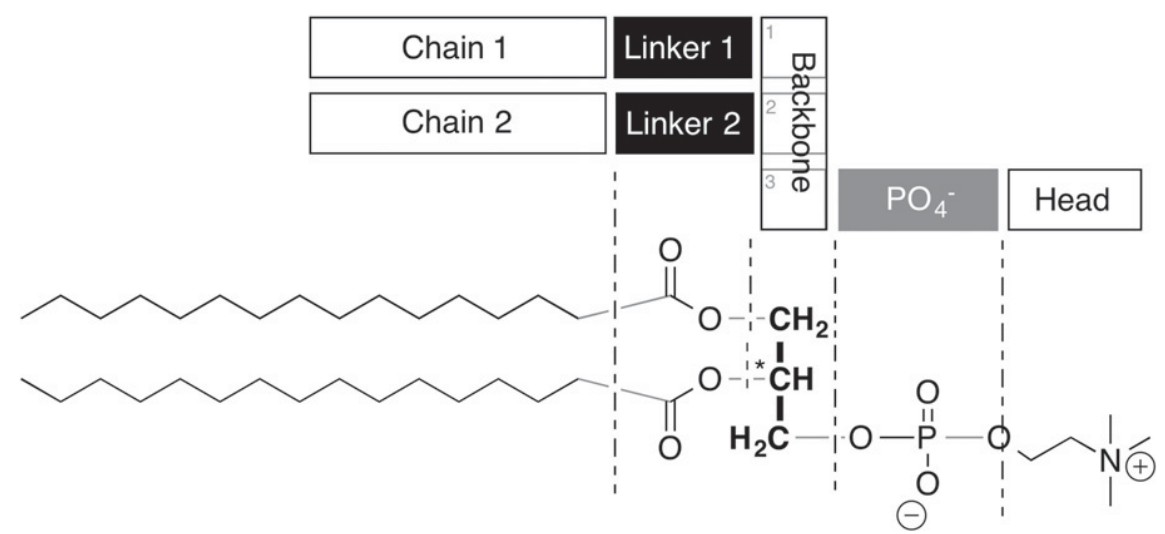

Fig. 1. Analysis of the modular structure of a glycerophospholipid. The molecule consists of 7 interchangeable units arranged around a central glycerol backbone. The two chains form the hydrophobic part of the molecule. The 5 other units build the hydrophilic part.
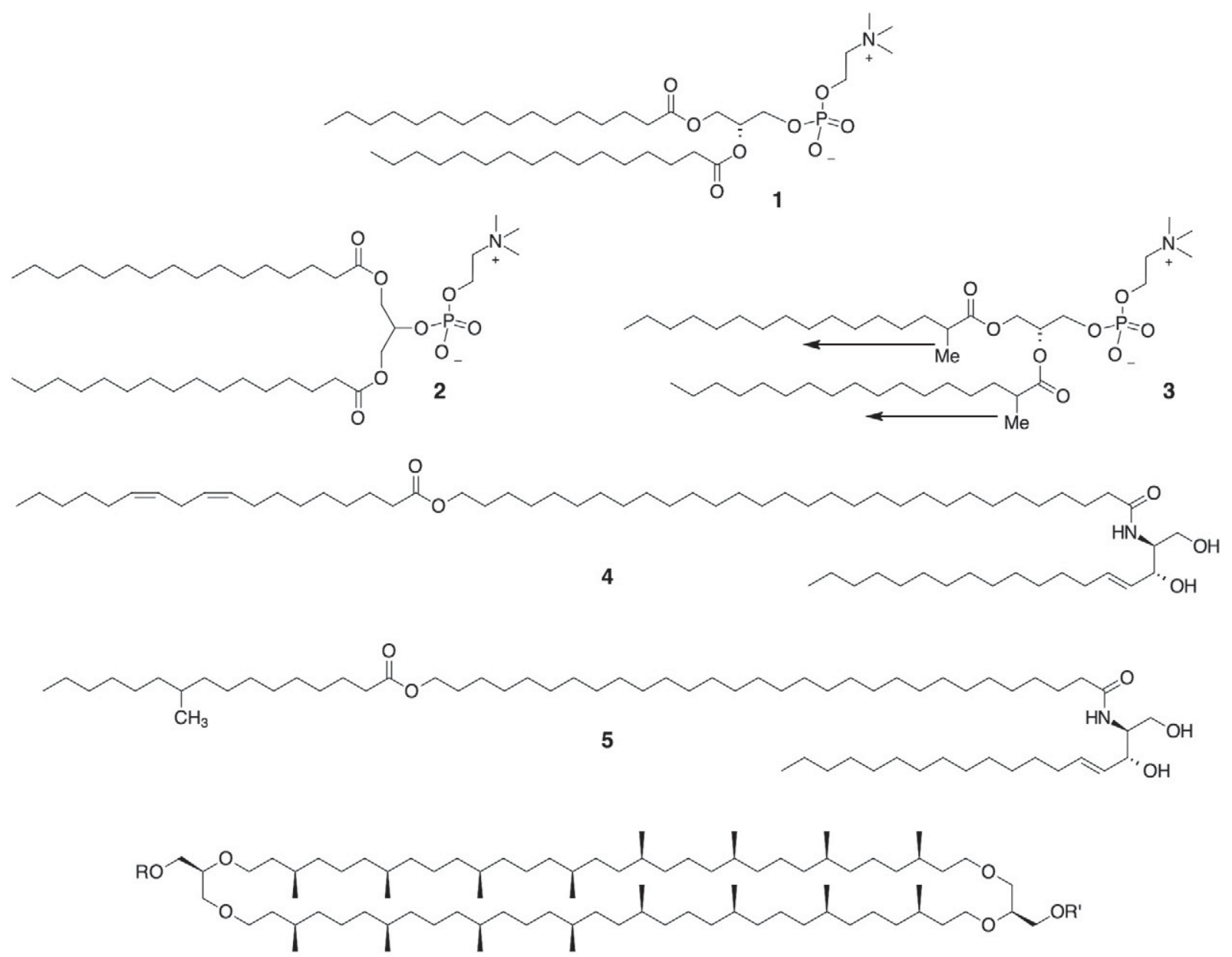

$$
6 \mathrm{R}=\mathrm{R}^{\prime}=\mathrm{H} \quad 7 \mathrm{R}=\mathrm{H}, \mathrm{R}^{\prime}=\mathrm{HOH}_{2} \mathrm{C}-\mathrm{C}(\mathrm{OH})-\left(\mathrm{CH}_{2} \mathrm{OH}\right)_{3}-\mathrm{CH}_{2} \mathrm{OH} \quad 8 \mathrm{R}=\mathrm{H}, \mathrm{R}^{\prime}=\mathrm{PO}(\mathrm{OH})_{2}
$$

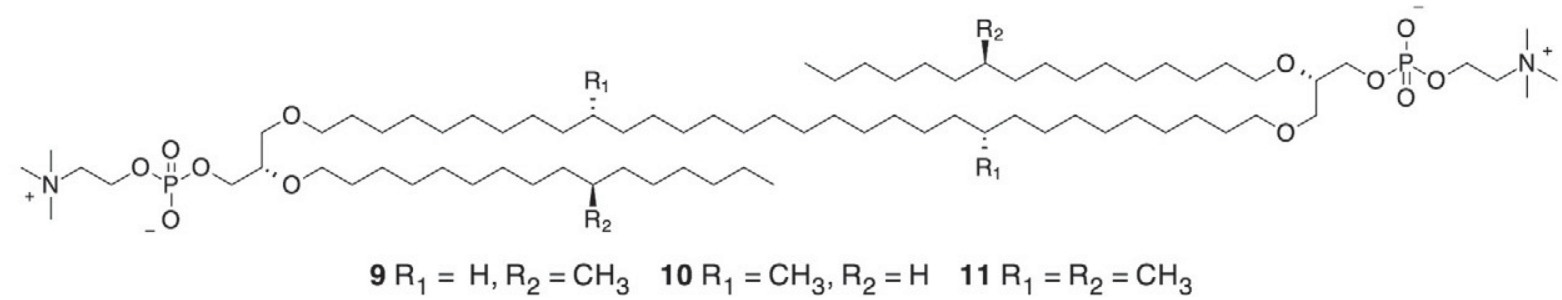

Fig. 2. Structure of a natural 1,2-diester phospholipids (1) compared to its 1,3-analog (2), a methyl-branched phosphocholine (3). Ceramid EOS (4) and its methyl-substituted ceramid 1 analog (5). Caldarchaeol (6), nonitolcaldarchaeol (7) and caldarcheolphosphate (8) as well as synthetic bolaphospholipids (9-11). 
in Fig. 1 is fully saturated and therefore contains no double bonds. Such a chain is abbreviated as C16:0 [6]. The head group of a natural phospholipid is typically a primary alcohol such as choline, ethanolamine, or even glycerol, or serine.

Each of these modules is exchangeable. And this leads to a large variety of molecules that is accessible to nature or chemists. E.g., the exchange of the ester group linking the hydrophobic tails with the glycerol backbone by an ether group leads to chemically more stable molecules and to changes in polymorphism. The hydrolysis reaction catalyzed by phospholipases $A_{1}$ and $A_{2}$ leading to a free fatty acid and a lysophospholipid can be prevented; a fact that might be important for using phospholipid vesicles as drug carriers in biological experiments. Through other synthetic modifications, small but significant changes can be introduced into the chemical structures leading to important changes of the phase behavior of phospholipids. This will lead to molecules that allow new insights into basic membrane biophysics properties.

\subsection{1,2-Phospholipids}

Four different phases have been found in fully hydrated 1,2-DPPC (see 1 in Fig. 2) being annealed for several days at $4{ }^{\circ} \mathrm{C}$ [7,8]. A subgel phase $L_{C}$ characterized by the packing of entire molecules forms only after several days at low temperature and transforms at $18.4{ }^{\circ} \mathrm{C}$ into an usual gel phase $\mathrm{L}_{\beta}$. The head group is only weakly hydrated in the subgel phase and occupies therefore less space leading to only a slight tilting of the chains. The gel phase of glycerophospholipids shows the typical orthorhombic packing of the acyl chains which are now strongly tilted to the bilayer normal. The head group contains around 15 water molecules in the hydration shell. The gel phase transforms into a socalled ripple phase $\mathrm{P}_{\beta^{\prime}}$ at $\mathrm{T}_{\mathrm{p}}=35.1{ }^{\circ} \mathrm{C}$ which melts at $\mathrm{T}_{\mathrm{m}}=41.1{ }^{\circ} \mathrm{C}$ into a lamellar $\mathrm{L}_{\alpha}$ phase with molten acyl chains (trans-gauche conformational change) exhibiting only a broad halo in the WAXS pattern.

The chain length has a strong influence on the phase behavior. The PCS with shorter chains (C10-C13) form very stable subgel phases which melt directly into the $\mathrm{L}_{\alpha}$ phase. The PCs with longer chains form the subgel phase only after long annealing times. The main phase transition from the liquid-crystalline $\mathrm{L}_{\alpha}$ phase into a gel phase does not show a pronounced hysteresis whereas the transition from the ripple into the normal gel phase can be markedly super-cooled. Increasing chain length leads to a monotonous but non-linear increase of the main phase transition temperature. The existence region $\Delta \mathrm{T}\left(\Delta \mathrm{T}=\mathrm{T}_{\mathrm{m}}-\mathrm{T}_{\mathrm{p}}\right)$ of the ripple phase decreases with an increase in chain length.

\subsection{1,3 Phospholipids}

A first artificial lipid system is accessible if the 1,2-linked chains are moved further apart to give a 1,3-constitutional isomer (see 2, Fig. 2). The 1,3-diester lipids with identical chains are symmetrical and achiral, unlike the natural 1,2-diester lipids. This lack of the stereo-center at the $s n-2$ carbon is the key for the synthesis of 1,3-diester lipids and these lipids can be synthesized from simple glycerol [9]. A substitution with 2 equivalents of an activated fatty acid ester leads to a 1,3-diester that can be readily transformed into a phospholipid.

Primary alcohols are more nucleophilic than secondary alcohols. A 1,3-diacyl substituted glycerol therefore would represent a thermodynamically more stable form than a 1,2-diacyl substituted glycerol. If the $s n-3$ alcohol is not protected by a phosphate diester, an acyl shift of the $s n-2$ fatty acid is found under acidic conditions [10]. This rare case is found in natural membranes: lysobisphosphatidic acid is accumulating in late endosomes at very high concentrations. The molecule with its unusual constitution can switch between a 2,2' and 3,3' acyl position geometry and therefore changes its overall structure. The shift occurs under acidic conditions found in the late endosomal pathway and might explain the fusogenicity of lysobisphosphatidic acid [11].

The Seeligs have found that for PC, PE, and PG lipids the corresponding head groups are aligned almost parallel to the bilayer horizon [12].
The glycerol backbone itself is however aligned perpendicular to it, in liquid-crystalline and gel state membranes as well as single crystals. 11-16 water molecules in the primary hydration shell, push the head group of PC $30^{\circ}$ away from the membrane surface. This tilt can be compensated by a negatively charged ion in the lipid surrounding solution.

Unlike 1,2-DPPC, the 1,3-DPPC shows a backbone orientation that runs parallel to the membrane horizon. However, the two carbonyl functions in 1,3-DPPC (2) do not have the same environment as shown by infrared spectroscopy [13]. The phosphate group shows a preferential tilting towards the $s n-1$ carbonyl. 1,3-DPPC (2) also shows a $\mathrm{T}_{\mathrm{m}}$ of $37^{\circ} \mathrm{C}$, which is $4{ }^{\circ} \mathrm{C}$ lower than that of 1,2 -DPPC (1). The 1,3-isomer probably shows less perfect packing, which is compensated by a tilt in the $\mathrm{L}_{c}$ phase.

Three different lamellar phases have been found in aqueous dispersions of 1,3-DPPC (2) by X-ray diffraction studies: (i) below $18{ }^{\circ} \mathrm{C}$, a hydrated "crystalline" bilayer phase, $\mathrm{L}_{\mathrm{c}}$, with a bilayer periodicity $\mathrm{d}=58 \AA \AA$; (ii) between $30{ }^{\circ} \mathrm{C}$ and $35{ }^{\circ} \mathrm{C}$, a more hydrated but interdigitated gel phase, with hexagonal chain-packing and $\mathrm{d}=47$ Å and (iii) above $37^{\circ} \mathrm{C}$, a highly hydrated liquid-crystalline $\mathrm{L}_{\alpha}$ phase with $\mathrm{d}=65 \AA$ A. The hydrocarbon chain interdigitation is deduced from the small bilayer periodicity, a sharp, symmetric wide-angle reflection at $\mathrm{s}=0.238 \AA^{-1}$, and an in-plane area per 1,3-DPPC at the interface of $\sim 80 \AA^{2}$. Nuclear magnetic resonance and neutron diffraction studies have suggested that the glycerol backbone of 1,3-DPPC 2 orients parallel to the bilayer surface, in contrast to its orientation in 1,2-DPPC 1 . The 1,3 conformation therefore leads to an increased intramolecular chain separation. The area requirement mismatch between the hydrophobic and hydrophilic parts of the molecule is now dominated by the interfacial region (the glycerol backbone).

We have recently reported 1,3-phospholipids containing amides replacing the common ester linkers [14]. Large extruded unilamellar vesicles assembled from these molecules turned out to be of a lenticular shape [15]. Moreover, the vesicles were mechanosensitive: a dye loaded into the aqueous lumen of the vesicles would not be released if the liposomes were left standing untouched. As soon as the vesicles were shaken, and therefore mechanically stimulated, the vesicle cargo was released. On the other hand, giant unilamellar vesicles made from 1,3-diamidophospholipids showed various geometries, faceted vesicles

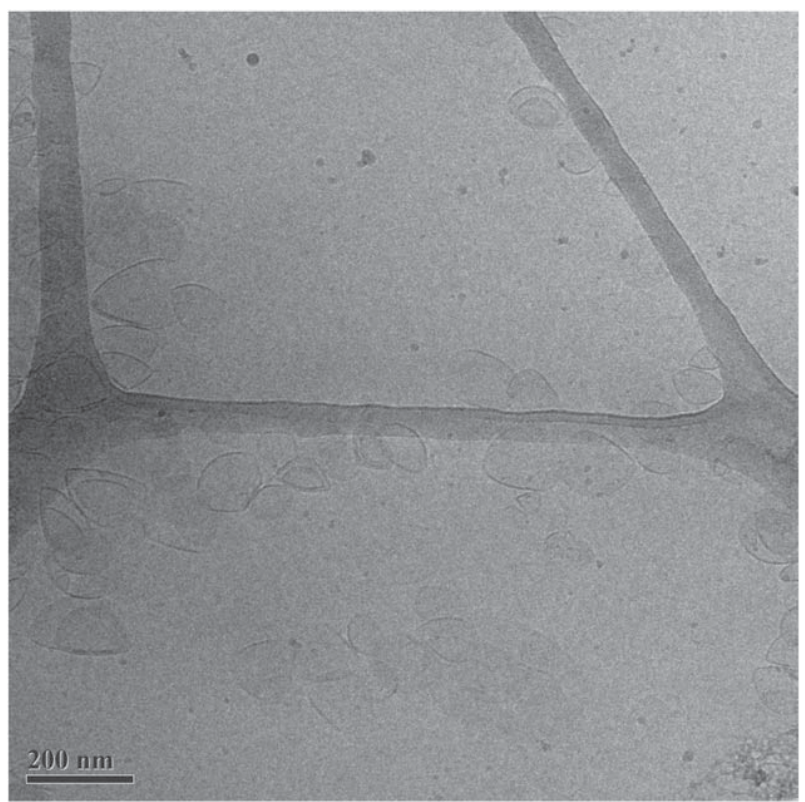

Fig. 3. Cryo-transition micrograph of faceted 1,3-diamido phospholipid vesicles after extrusion through a $100 \mathrm{~nm}$ membrane. The bilayer is not continuous but shows facets and edges, calling for defects in the membrane structure. The picture was taken by Dr. T. Ishikawa. 
in particular. And we were tempted to call the vesicle self-assembly process "vesicle origami" (see Fig. 3 ).

Intrigued by these effects we have started to analyze the mechanosensitivity effect in more detail. Additionally, the new compounds have been investigated in thin layers confined to the surface of water by highly surface sensitive techniques such as infrared reflexionabsorption spectroscopy (IRRAS) and grazing incidence X-ray diffraction (GIXD) [16]. GIXD revealed a unique, homogeneous structure for the longer-chain compounds at different lateral pressures. The existence of strong head group interactions due to the formation of a hydrogen bond network was clearly revealed by IRRAS and by the high rigidity of the layers. This rigidity is correlated to the marginal change of the chain tilt upon compression of the monolayer (see Fig. 4). Surprisingly, the tilt angle variation is similar to that of a standard glycero-phospholipid as DSPC [17] showing the strong influence of the van der Waals interactions between the hydrophobic chains on the packing properties in thin layers confined at the air/water interface. The strong hydrogen-bonding interactions between the head groups of 1,3-diamidophospholipids could be also responsible for the observed facetted shape in 3D aggregates. Such facets and the corresponding edges place high demands on the membrane bilayer physics. Following a classification of lamellar phases, the formed bilayer would probably not be continuous (type 1) but be of type 2, i.e. needing defects in order to react to the imposed curvature constraints [18]. If it would be possible to control type 2 bilayer membranes, new phospholipid soft matter geometries, vesicle origami, could become feasible. The attenuation of membrane defects is hypothesized to be the origin of shear-sensitive liposomes [15].

A clear difference between bilayer and monolayer stabilizations was found with the main phase transition temperature of the bilayer being significantly higher than the extrapolated critical temperature (the temperature above which the monolayer cannot be compressed into the condensed state) of the monolayer [16]. A plausible explanation - for this effect could be phospholipid chain interdigitation that was possible by spacing the lipid tails in a 1,3-instead of a 1,2-fashion. This was raising the more general question about the requirements for bilayer membrane interdigitation that will be answered in the following paragraphs.

\subsection{Interdigitation}

1,2-Diacyl-P-O-ethylphosphatidylcholines are a novel class of ationic lipids, which are synthesized by esterification of phosphatidylcholines with ethyl-trifluoromethylsulfonates [19]. Some derivatives have already shown promising properties as liposomal

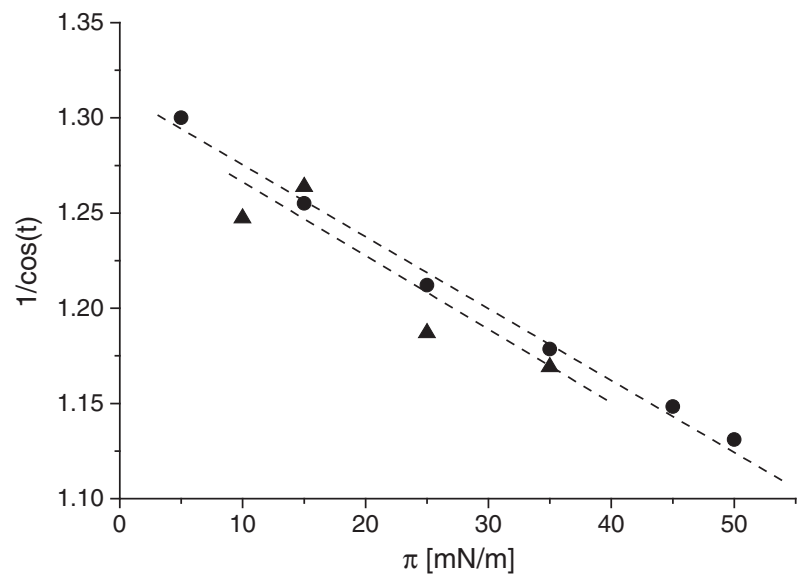

Fig. 4. $1 / \cos (t)$ versus temperature. The tilt angle $t$ of the aliphatic chains of 1,3-diamido C18 phospholipid $(\boldsymbol{\Delta})$ and DSPC $(\bullet)$ monolayers at $20{ }^{\circ} \mathrm{C}$ determined by GIXD experiments. The linear extrapolation towards zero tilt angle $(1 / \cos (t)=1)$ yields the pressure of the tilting transition. The non-tilted phase can be expected only at unattainable high pressure values.
DNA transfection agents. It was found that these derivatives form a chain interdigitated gel phase. Only few phosphatidylcholine lipids form spontaneously interdigitated lamellar gel phases, e.g., 1,2-dihexadecylphosphatidylcholine (DHPC) [20], the ether analog of 1,2-dipalmitoylphosphatidylcholine (DPPC), 1-palmitoyl-2-(16fluoropalmitoyl)-phosphatidylcholine [21], a fluorinated analog of DPPC, and 1,3-DPPC [22].

In this interdigitated gel phase, the hydrocarbon chain packing is rather compact, whereas the head groups are more loosely packed than in non-interdigitated gel phases. The presence of the additional ethyl group at the phosphate moiety increases the volume of the polar head group. Therefore, interdigitation of the chains leads to a larger separation of the head groups and releases the stress arising from steric crowding and electrostatic repulsion of the positively charged head groups. The main transition leads to a non-interdigitated $\mathrm{L}_{\alpha}$ phase seen by a sharp increase in $d$ spacing. This increase is however mostly due to an increase of the water layer thickness between the bilayers since the decrease in the hydrocarbon layer thickness due to melting of the chains compensates the increase by de-interdigitation.

Small changes in the chemical constitution of the backbone region can lead to conformational changes [23]. The exchange of a DPPC diester with C16:0et-C16:0et-PC, a diether analog, induces chain interdigitation, and increases the polarity of the head group. Changing the glycerol backbone to an ether substitution pattern also changes the backbone away from its orientation perpendicular to the membrane horizon. This induces an increased intermolecular spacing. Less sterical crowding in the head group leads to more water incorporated in the hydrophilic region. This, together with fully extended hydrocarbon chains, leads to an increase in $\mathrm{T}_{\mathrm{m}}$.

In general, for phospholipids with an $\mathrm{CH}_{2}$ difference $>4$ between the two chains an interdigitation is found. For phospholipids with a large difference between tails (SDPC [C18:0 and C10:0] or SLPC [C18:0 and C12:0]) this leads to a mixed interdigitation [24] in the gel phases at $\mathrm{T}<\mathrm{T}_{\mathrm{m}}$ : in a bilayer, the two shorter chains touch end-to-end. The longer tails fully reach into the opposite bilayer half. This leads to tight tetragonal or orthorhombic crystal packing where the individual lattice points are not exchangeable. A different type of interdigitation is found for SMPC [C18:0 and C14:0] where the longer chains do not reach into the opposite bilayer half but form a fluidintermediate state in the hydrophobic core of the membrane (see Fig. 5).

This fluid intermediate had been proposed by Stümpel, Nicksch and Eibl [25]. They also saw that changes in tail-length at sn-2 position have more important effects on $\mathrm{T}_{\mathrm{m}}$ than changes in $s n-1$. The longer chains at $s n-1$ end up in the fluid region of the membrane and therefore do not contribute anymore to the $\Delta H$ change.

Fatty acyl chain interdigitation can be introduced by spacing the head groups apart a bit further, e.g., by going from a negatively charged phosphate diester to a neutral phosphate triester [26]. In the case of diC14-amidine one extra $\mathrm{CH}_{2}$ atom in the backbone is thought to space the tails apart a bit more than natural, leading to an ease of chain interdigitation [27].

\subsection{Influence of the head group}

The bulky and strongly hydrated head group determines the molecular area in phosphatidylcholine monolayers and bilayers. For example, a DPPC 1 monolayer at the air/water interface forms a rectangular in-plane lattice with strongly tilted acyl chains $\left(\sim 30^{\circ}\right.$ at a lateral pressure of $30 \mathrm{mN} / \mathrm{m}$ ). The corresponding gel phase in multilamellar dispersions is a lamellar $\mathrm{L}_{\beta^{\prime}}$ phase with tilted chains. The chain tilt is the same in monolayers and bilayers at a comparable lateral pressure $(\sim 30 \mathrm{mN} / \mathrm{m})$. The reason for the chain tilt is the area mismatch between the head group and two ordered acyl chains. To increase their van der Waals interactions the chains have to be tilted. The reduction of the effective head group area leads to a decrease of 

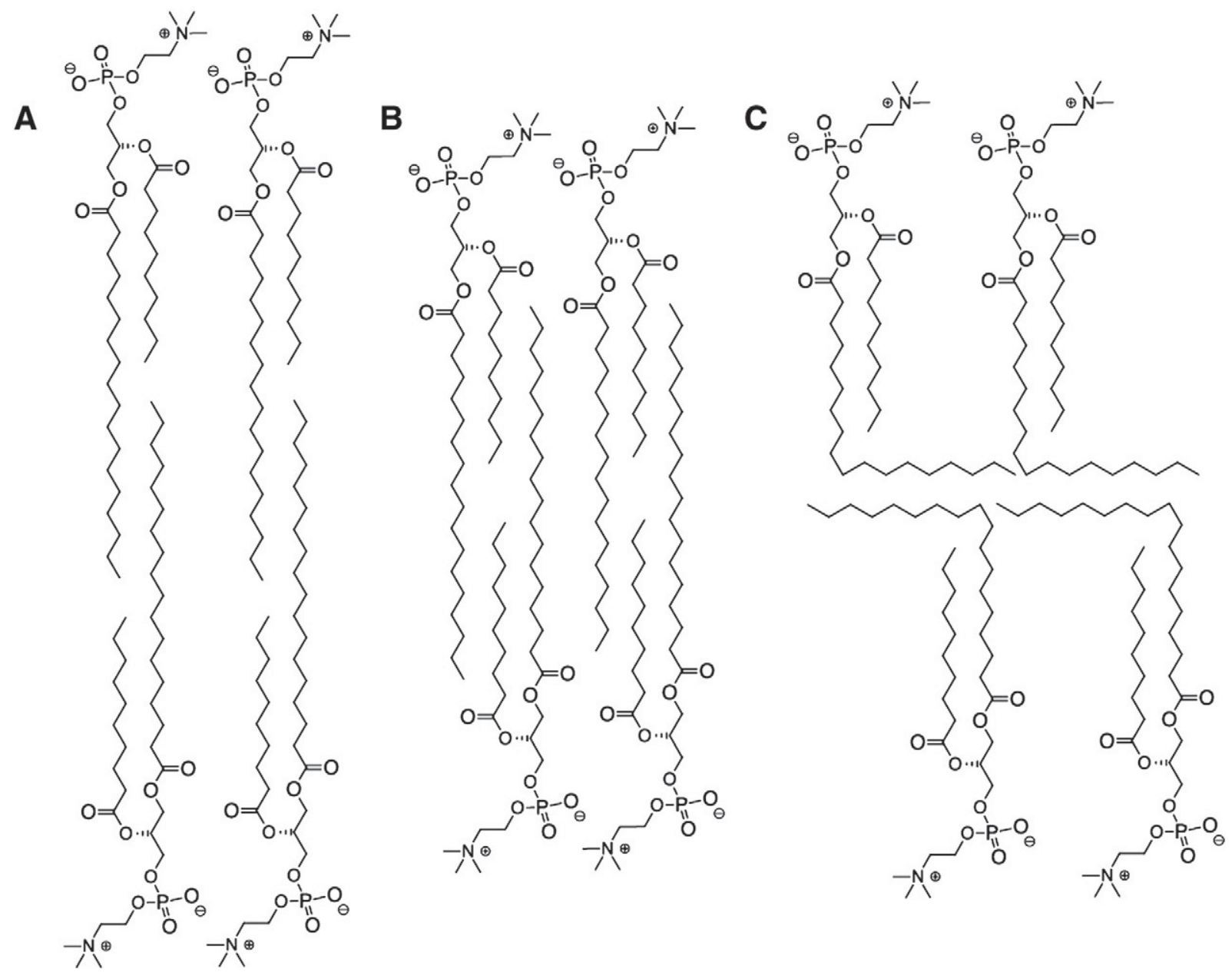

Fig. 5. Different interdigitation models: A) match of acyl chain lengths, B) complete interdigitation, and C) introduction of a fluid-like layer in the hydrophobic core of the bilayer.

the tilt angle. This can be obtained by changing the hydration shell around the head group or by changing the head group conformation. In the case of phosphatidylcholines, the head groups are oriented nearly parallel to the water surface [28]. The orientation change to a more vertical arrangement leads to the reduction of the area requirement mismatch. Such a behavior was found, e.g., for the binding of an enzyme (phospholipase $\mathrm{A}_{2}$ ) to a S-DPPC monolayer. The protein binding enforces a dehydration and reorientation of the PC head group $[29,30]$. This process is highly cooperative. Another possibility to reduce the tilt angle is the insertion of alkanes into the hydrophobic part of the monolayer [31]. In this case, the effective head group area remains unchanged, but the alkanes can be incorporated into the ordered lipid arrays leading to increased van der Waals interaction between non-tilted chains.

A third possibility is a partial dehydration of the head group as observed for a subphase containing alcohol [32]. However, results obtained with monolayers and foam films are in contrast to those obtained with lipid multilayers. GIXD experiments have shown that the effective area per head group decreases with an increase in ethanol concentration leading to a reduction of the chain tilt angle. The influence of ethanol on self-assembled aggregates of phospholipid molecules has been reported in [33-39]. In these investigations, lipid bilayers dispersed as unilamellar or multilamellar vesicles in an aqueous solution have been used. It was found that the addition of ethanol first increases the tilt angle of the chains. Ethanol molecules bound to a phospholipid head group start to increase the area per lipid molecule in the interfacial region from $0.48 \mathrm{~nm}^{2}$ in pure water to $0.67 \mathrm{~nm}^{2}$ in $0.4 \mathrm{M}$ ethanol due to a substitution of water molecules from the hydration shell of the head group by ethanol. The hydrocarbon tilt is enlarged by up to $23^{\circ}$ (from $30^{\circ}$ to $53^{\circ}$ ) to allow the chains to maintain a constant packing density in the hydrocarbon layer. At larger ethanol concentrations, the strongly tilted phase becomes unstable and transforms into a phase with interdigitated acyl chains. In the case of 1,2-DPPC 1, the interdigitated phase appears at $50 \mathrm{mg}$ ethanol per $\mathrm{ml}$ solution.

\subsection{Influence of fatty chain modifications}

One way to keep the membrane fluid enough is the incorporation of phospholipids with shorter fatty acid chains exhibiting a lower transition temperature from the gel to the liquid-crystalline state. However, nature itself more often uses unsaturated fatty acids, for instance oleic and linoleic acids and also fatty acids with additional double bonds [40]. The unsaturated fatty acids are mainly attached at the $s n-2$ position of the glycerol backbone of glycerophospholipids. The reason for the lower main transition temperature is the disordering effect on the packing properties induced by the double bond $[41,42]$. Another effect of double bonds in phospholipids, also as protection against oxidative stress, is to react with oxygen resulting in a degradation of the fatty acid and therefore of the phospholipid. Unsaturated fatty acids mainly bound to the $s n-2$ position of glycerolipids can be easily deacylated by phospholipase $\mathrm{A}_{2}$ [43]. Higher unsaturated fatty acids, for instance arachidonic acid, can be hydrolyzed in this way followed by metabolization in two directions producing prostanoids and eicosanoids [44]. Membranes adjust their composition according to the environmental conditions. For example, bacteria alter their membrane 
lipid composition, and therefore the membrane fluidity, in response to changes in growth temperature [45].

It is interesting to note that the lipid systems become more disordered by decreasing the chain lengths. The area per chain observed at the same temperature is larger for lipids with shorter chains. This could be the expression of an incremental steric hindrance which finally leads to vesicles with completely uncorrelated bilayers. E.g., dioctanoylphosphatidylcholine (C8:0) forms micelles, dinonaoylphosphatidylcholine (C9:0) unilamellar vesicles, and the diacylphosphatidylcholines with chain lengths $>10$ form multilamellar vesicles [46].

Another mechanism to ensure membrane fluidity was found in the lipids of eubacteria and archaebacteria. From the lipids of eubacteria branched fatty acids were isolated, especially iso- and anteiso-methyl branched stearic and palmitic acids. The occurrence of branched fatty acids in general was published in an early summary [47,48]. At that moment the function of these compounds in the membrane was rather speculative.

The influence of iso-and anteiso methyl branching on the physicalchemical properties was first investigated by the McElhany group using 1,2-diacyl-sn-glycerophosphocholines (1,2-di-Cn:0-PC, with $\mathrm{n}$ as the number of carbon atoms in one chain) [49,50]. The lipids showed continuous decrease of the $\mathrm{T}_{\mathrm{m}}$-values starting from unbranched via iso-branched to the anteiso-branched 1,2-dipalmitoyl-sn-glycero-3phosphocholine. We have synthesized a series of 1,2-diacyl-sn-glycero3-phosphocholines with racemic methyl branched fatty acids in which the methyl group is moving along the main chain (see $\mathbf{3}$, Fig. 2) [51,52]. Beside the common occurrence of iso- and anteisomethyl branched fatty acids, the compounds with methyl groups in other positions are rarely found in nature. The position of the methyl branching has a dramatic effect on the $\mathrm{T}_{\mathrm{m}}$. Already a methyl group in the 2-position of the main fatty acid chain leads to a decrease of $\mathrm{T}_{\mathrm{m}}$ - connected with a smaller transition enthalpy compared to lipids with unbranched chains. Moving this methyl branch step by step to the end of the main chain leads to a deep minimum in the phase transition temperature. Phospholipids with two 7- or 8-methylhexadecanoic acid residues show even no phase transition in the experimentally accessible range. One could imagine that the methylbranched PCs behave like the unbranched ones but with shorter main chains. A direct comparison between branched and unbranched 1,2-diacylglycero-3-phosphocholines indicates that this scenario is qualitatively correct, however the main phase transition temperatures are not exactly the ones of the corresponding PCs with shorter chains but higher (see Fig. 6). Therefore, the packing of such methyl-branched PCs has to be optimized in another way. SAXS experiments show the

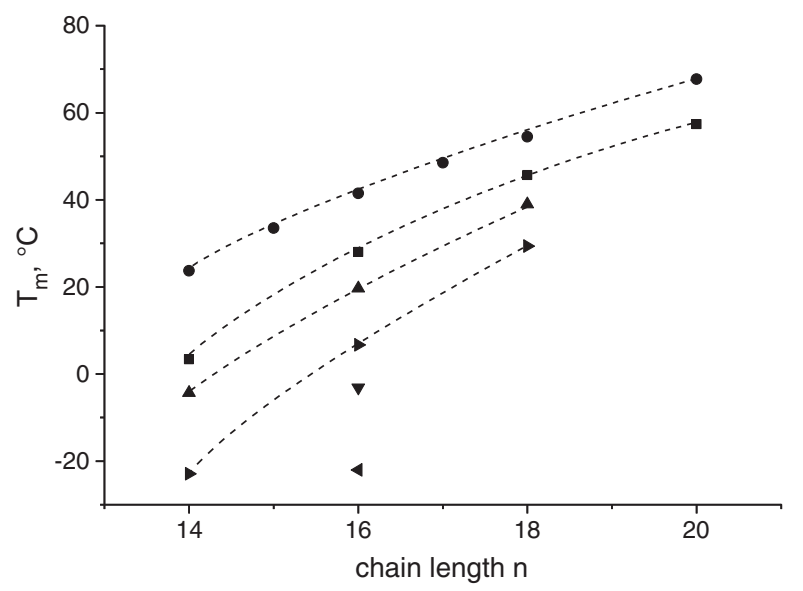

Fig. 6. Main phase transition temperatures $T_{m}$ of $1,2-d i-C n: 0-P C(\bullet)$ and methyl branched PCs 1,2-di-(xC1-Cn:0)-PC with $\mathrm{x}$ as the position of the methyl $\mathrm{C} 1$ branches $(\boldsymbol{\square}-\mathrm{x}=2, \boldsymbol{\Delta}-\mathrm{x}=3, \boldsymbol{-} \mathrm{x}=4, \boldsymbol{\nabla}-\mathrm{x}=5,-\mathrm{x}=6)$ versus the chain length $\mathrm{n}$. appearance of an interdigitated gel phase with the introduction of methyl branches in 2-position.

Another example for the action of methyl branches in a fatty acid chain are ceramides. The general structure of these essential natural products consists on the one hand of a sphingosin (minor amounts of sphinganin were found), phytosphingosine and 6-hydroxysphingosine and on the other hand of fatty acids bound via amide bond to the nitrogen atom of the sphingobases (for a summary see [53]). The most outstanding structures are the ceramides EOS (4) and EOP. There is still a controversial discussion about the role of these ceramides. The synthesis is not easy regarding the blocking group strategy. However, the synthesis was described by Mori and coworkers [54]. To find a more stable derivative of ceramide EOS the sensitive and oxidable linoleic acid was replaced by the racemic 10-methyl palmitic acid (5). The simplification of the synthetic procedure consists in the fact that triacontan-1,30-diol could be acylated with 10-methyl-palmitic acid chloride to yield the monoester [55]. This compound was oxidized to the corresponding $\omega$-ester of triacontanoic acid which was bound to the amino group of sphingosine. The EOS derivative demonstrates that the exchange of linoleic acid for the branched fatty acid leads to nearly the same phase transition temperature $\left(85^{\circ} \mathrm{C}\right.$ versus $79^{\circ} \mathrm{C}$, respectively). Whereas the $\mathrm{T}_{\mathrm{m}}$ of natural EOS tends to shift to slightly higher $\mathrm{T}_{\mathrm{m}}$ values during consecutive heating cycles, the phase transition temperature of the branched chain EOS derivate is constant. In summary, the branching in the EOS molecule has a similar effect on the packing properties as discussed above. Only one chain segment contributes to the packing and therefore to the main phase transition temperature. Depending on the position of the methyl branch this can be the part between the branched C-atom and the chain end if the gel phase is an interdigitated one, or the part between the carboxylic group and the branching if the position of the methyl group is too close to the chain end. In the latter case, an interdigitation does not sufficiently improve the attractive interactions between the chains. An additional advantage of the branched EOS derivative is its stability not only during the synthesis.

\subsubsection{The influence of methyl-branched chains in bolaamphiphiles}

Archaebacteria occupy ecological niches like saline lakes, strict anaerobic conditions or in hot geysers and acidic milieu [56,57]. In this case, the hydrophobic residues are connected to the glycerol moiety via ether bonds. In addition, the ether bonds are formed in the inverse 2,3-sn-configuration (6) and the hydrophobic chains contain methyl branches in the so called isoprene rhythm and in some forms also cyclopentyl rings. Whereas the ether bonds and the inverse stereochemistry are necessary for the stability against enzymes and hydrolytic conditions, the methyl branches and cyclopentyl rings are responsible for membrane flexibility [58-60].

Archaebacterial lipids, especially those with membrane spanning hydrophobic chains found in the methanogenes and thermoacidiphiles are of great interest in biotechnology, material sciences and pharmacy. Due to the bipolar character of these lipids they are able to form monolayer membranes also called archeosomes. However, the isolation of well-defined lipids from natural sources is very difficult. Therefore chemical synthesis is the method of choice. But total synthesis of archaebacterial lipids is not an easy task. So chemists have verified the complex structure. A lot of preparative work was done over the last years to create new and simpler structures for the general characterization of the unusual aggregation behavior of these bolaamphiphiles and also for the preparation of stable liposomes. A good summary over the last trends in this field was given in by Meister and Blume [61].

In this context the question arose in our group whether methyl branches in the isoprene rhythm, as used by nature in the biosynthesis of terpenes [60], are needed to model the hydrophobic chain of archaebacterial lipids.

Based on the results obtained with 1,2-diacyl-PCs [51,52], showing the influence of the position of the methyl branches in the main chains 
on the main phase transition temperature, the idea developed that a substitution in the most disturbing $10,10^{\prime}$-positions in a membrane spanning chain and/or in the 10-position in the C16-chain of our tetraether model lipids (structures 9-11, Fig. 2) can mimic the natural bipolar lipids [62]. Already the introduction of only one methyl group in 10-position of the two $\mathrm{C}_{16}$ chains lowers the phase transition from $61{ }^{\circ} \mathrm{C}$ for an unbranched model compound to $17{ }^{\circ} \mathrm{C}$. When the two methyl groups are in the 10 and $10^{\prime}$-position of the membrane spanning chain, a $\mathrm{T}_{\mathrm{m}}$-value of $9{ }^{\circ} \mathrm{C}$ was found.

With 4 methyl groups (11) no phase transition was found above $0{ }^{\circ} \mathrm{C}$. This is in accordance with the $\mathrm{T}_{\mathrm{m}}$ values found for natural archaebacterial lipids [63]. The new model compounds form closed lipid vesicles as a freeze fracture replica electron microscopy study has shown.

\section{Conclusions}

Synthetic analogs of natural lipids are valuable tools to assess biophysical properties of monolayer and bilayer membranes. For decades, the interdisciplinary field has united chemists and physicists and generated knowledge that is of high importance for biology and medicine alike. Using synthetic lipids modified in the different parts of the molecule in a defined way, much information was obtained about the interplay of different forces determining the structures formed in aqueous dispersions of such lipids. The competing forces could be tuned to understand their importance for interactions with other biomolecules. Not only the different sizes of the hydrophilic and hydrophobic parts of the molecules led to different superstructures but also the ability to form additional strong intermolecular interactions, e.g., a hydrogen bonding network between head groups.

Based on chemical modifications found in nature, the synthesis of new lipids led to new materials with interesting properties. The extension of the described principles to other amphiphilic compounds seems to be very promising.

\section{Acknowledgments}

AZ thanks the Swiss National Science Foundation (PP00P2_138926/1, NCCR Chemical Biology, NRP62) and the University of Fribourg for funding.

\section{References}

[1] Vereb G, Szöllosi J, Mastko J, Nagy P, Farkas T, Vigh L, et al. Dynamic, yet structured: the cell membrane three decades after the Singer-Nicholson model. Proc Natl Acad Sci U S A 2003;100:8053-8.

[2] Aplin AE, Howe A, Alahari SK, Juliani RL. Signal transduction and signal modulation by cell adhesion receptors: the role of integrins, cadherins, immunoglobulin-cell adhesion molecules, and selectins. Pharmacol Rev 1998;50:197-263.

[3] Vaz WLC, Goodsaid-Zalduondo F, Jacobson K. Lateral diffusion of lipids and proteins in bilayer-membranes. FEBS Lett 1984;174(2):199-207.

[4] Jacobson K, Ishihara A, Inman R. Lateral diffusion of proteins in membranes. Annu Rev Physiol 1987;49:163-75.

[5] Bagatolli LA, Mouritsen OG. Is the fluid mosaic (and the accompanying raft hypothesis) a suitable model to describe fundamental features of biological membranes? What may be missing? Front Plant Sci 2013;4:457.

[6] Mouritsen OG. Life-as a matter of fat: the emerging science of lipidomics. Berlin: Springer; 2005.

[7] Nagle JF, Tristram-Nagle S. Structure of lipid bilayers. Biochim Biophys Acta 2000;1469:159-95.

[8] Matsuki H, Goto M, Tada K, Tamai N. Thermotropic and barotropic phase behavior of phosphatidylcholine bilayers. Int J Mol Sci 2013;14:2282-302.

[9] Fedotenko IA, Holme MN, Tanasescu R, Zaffalon P-L, Zumbuehl A. Putting the "P" into phospholipids. Chimia 2011;65:859-62.

[10] Andresen TL, Davidsen J, Begtrup M, Mouritsen OG, Jørgensen K. Enzymatic release of antitumor ether lipids by specific phospholipase A2 activation of liposome-forming prodrugs. J Med Chem 2004;47:1694-703.

[11] Goursot A, Mineva T, Bissig C, Gruenberg J, Salahub DR. Structure, dynamics, and energetics of lysobisphosphatidic acid (LBPA) isomers. J Phys Chem B 2010;114:15712-20.

[12] Seelig A, Seelig J. Membrane structure encyclopedia of physical science and technology3rd ed. ; 2002.
[13] Dluhy RA, Chowdhry BZ, Cameron DG. Infrared characterization of conformational differences in the lamellar phases of 1,3-dipalmitoyl-sn-glycero-2-phosphocholine. Biochim Biophys Acta 1985;821:437-44.

[14] Fedotenko IA, Zaffalon P-L, Favarger F, Zumbuehl A. The synthesis of 1,3diamidophospholipids. Tetrahedron Lett 2010;51:5382-4.

[15] Holme MN, Fedotenko IA, Abegg D, Althaus J, Babel L, Favarger F, et al. Shear-stress sensitive nanocontainers for targeted drug delivery. Nat Nanotechnol 2012;7:536-43.

[16] Fedotenko IA, Stefaniu C, Brezesinski G, Zumbuehl A. Monolayer properties of 1,3diamidophospholipids. Langmuir 2013;29:9428-35.

[17] Ropers M-H, Brezesinski G. Lipid ordering in planar 2D and 3D model membranes. Soft Matter 2013;9:9440-8.

[18] Dubois M, Zemb T. Swelling limits for bilayer microstructures: the implosion of lamellar structure versus disordered lamellae. Curr Opin Colloid Interface Sci 2000;5:27-37.

[19] MacDonald RC, Rakhmanova VA, Choi KL, Rosenzweig HS, Lahiri MK. Oethylphosphatidylcholine A metabolizable cationic phospholipid which is a serum-compatible DNA transfection agent. J Pharm Sci 1999;88:896-904.

[20] Laggner P, Lohner K, Degovics G, Müller K, Schuster A. Structure and thermodynamics of the dihexadecylphosphatidylcholine water-system. Chem Phys Lipids 1987;44:31-60.

[21] Hirsh DJ, Lazaro N, Wright LR, Boggs JM, McIntosh TJ, Schaefer J, et al. A new monofluorinated phosphatidylcholine forms interdigitated bilayers. Biophys J 1998;75:1858-68.

[22] Serallach EN, Dijkman R, de Haas GH, Shipley GG. Structure and thermotropic properties of 1,3-dipalmitoyl-glycero-2-phosphocholine. J Mol Biol 1983;170:155-74.

[23] Lewis RNAH, Pohle W, McElhaney RN. The interfacial structure of phospholipid bilayers: differential scanning calorimetry and Fourier transform infrared spectroscopic studies of 1,2-dipalmitoyl-sn-glycero-3-phosphorylcholine and its dialkyl and acyl-alkyl analogs. Biophys J 1996;70:2736-46.

[24] Hui SW, Mason JT, Huang C. Acyl chain interdigitation in saturated mixed-chain phosphatidylcholine bilayer dispersions. Biochemistry 1984;23:5570-7.

[25] Stuempel J, Nicksch A, Eibl H. Calorimetric studies on saturated mixed-chain lecithin-water systems. Nonequivalence of acyl chains in the thermotropic phase transition. Biochemistry 1981;20:662-5.

[26] Winter I, Pabst G, Rappolt M, Lohner K. Refined structure of 1,2-diacyl-P-Oethylphosphatidylcholine bilayer membranes. Chem Phys Lipids 2001;112:137-50.

[27] Rozenfeld JHK, Duarte EL, Oliveira TR, Lonez C, Ruysschaert J-M, Lamy MT. Oligonucleotide adsorption affects phase transition but not interdigitation of diC14-amidine bilayers. Langmuir 2013;29:11102-8.

[28] Brumm T, Naumann C, Sackmann E, Rennie AR, Thomas RK, Kanellas D, et al. Conformational changes of the lecithin headgroup in monolayers at the air/water interface. A neutron reflection study. Eur Biophys J 1994;23:289-95.

[29] Dahmen-Levison U, Brezesinski G, Möhwald H. Specific adsorption of PLA(2) at monolayers. Thin Solid Films 1998;329:616-20.

[30] Möhwald H, Dahmen U, de Meijere K, Brezesinski G. Lipid monolayers to understand and to control properties of fluid interfaces. Prog Colloid Polym Sci 1998;109:3-12.

[31] Brezesinski G, Thoma M, Struth B, Möhwald H. Structural changes of monolayers at the air/water interface contacted with n-alkanes. J Phys Chem 1996;100:3126-30.

[32] Brezesinski G, Müller HJ, Toca-Herrera JL, Krustev R. X-ray diffraction and foam film investigations of PC head group interaction in water/ethanol mixtures. Chem Phys Lipids 2001;110:183-94

[33] McIntosh TJ, McDaniel RV, Simon SA. Introduction of an interdigitated gel phase in fully hydrated phosphatidylcholine bilayers. Biochim Biophys Acta 1983;731:109-14.

[34] Rowe ES, Cutrera TA. Differential scanning calorimetric studies of ethanol interactions with distearoylphosphatidylcholine: transition to the interdigitated phase. Biochemistry 1987;29:10398-404.

[35] Vierl U, Löbbecke L, Nagel N, Cevs G. Solute effects on the colloidal and phase behavior of lipid bilayer membranes: ethanol-dipalmitoylphosphatidylcholine mixtures. Biophys J 1994;67:1067-79.

[36] Adachi T, Takahashi H, Ohki K, Hatta I. Interdigitated structure of phospholipidalcohol systems studied by X-ray diffraction. Biophys J 1995;68:1850-5.

[37] Komatsu H, Okada S. Ethanol-induced aggregation and fusion of small phosphatidylcholine liposome: participation of interdigitated membrane formation in their processes. Biochim Biophys Acta 1995;1235:270-80.

[38] Löbbecke L, Cevc G. Effects of short-chain alcohols on the phase behavior and interdigitation of phosphatidylcholine bilayer membranes. Biochim Biophys Acta 1995;1237:59-69.

[39] Ho C, Stubbs CD. Effect of n-alcanols on lipid bilayer hydration. Biochemistry 1997;36:10630-7.

[40] Fajardo VA, McMeekin L, LeBlanc PJ. Influence of phospholipid species on membrane fluidity: a metaanalysis for a novel phospholipid fluidity index. J Membr Biol 2011;244(2):97-103.

[41] Cevc G. How membrane chain-melting phase transition temperature is affected by the lipid chain asymmetry and degree of unsaturation. An effective chain-length model. Biochemistry 1991;30:7186-93.

[42] Barton PG, Gunstone FD. Hydrocarbon chain packing and molecular motion in phospholipid bilayers formed from unsaturated lecithins. J Biol Chem 1975;250:4470-6.

[43] Murakami M, Taketomi Y, Sato H, Yamamoto K. Secreted phospholipase A2 revisited. J Biochem 2011;150(3):233-55.

[44] Fanning LB, Boys JA. Lipid mediators and allergic diseases. Ann Allergy Asthma Immunol 2013;111:155-62.

[45] Hänel F, Brezesinski G, Dörfler H-D, Hilliger M, Gräfe U. Thermal regulation of phospholipid fluidity in Cornybacterium glutamicum. J Basic Microbiol 1986;26:409-13.

[46] Racey TJ, Singer MA, Finegold L, Rochon P. The influence of fatty acyl chainlength and head group on the size of multilamellar vesicles. Chem Phys Lipids 1989;49:271-88. 
[47] Kaneda T. Fatty-acids of genus Bacillus - example of branched-chain preference. Bacteriol Rev 1977; 41:391-418.

[48] Russel NJ, Fukunaga N. A comparison of thermal adaptation of membrane lipids in psychrophilic and thermophilic bacteria. FEMS Microbiol Rev 1990;75:171-82.

[49] Silvius JR, McElhaney RN. Effects of phospholipid acyl chain structure on physicochemical properties. 1. Isobranched phosphatidylcholines. Chem Phys Lipids 1979;24:287-96.

[50] Silvius JR, McElhany RN. Effects of phospholipid acyl chain structure on thermotropic phase properties. 3. Phosphatidylcholines with (-)-anteiso and (+/-)-anteiso acyl chains. Chem Phys Lipids 1980;26:67-77.

[51] Nuhn P, Brezesinski G, Dobner B, Förster G, Gutheil M, Dörfler H-D. Synthesis calorimetry and X-ray diffraction of lecithins containing branched fatty acyl chains. Chem Phys Lipids 1986;39:221-36.

[52] Dörfler H-D, Brezesinski G, Nuhn P, Dobner B, Gutheil M. Thermal phase-transition of homologous alpha-methyl and beta-methyl branched lecithins in a water saturated 2-phase region. Colloid Polym Sci 1983;261:1033-7.

[53] Masukawa Y, Narita H, Shimizu E, Kondo N, Sugai Y, Oba T, et al. Characterization of overall ceramide species in human stratum corneum. J Lipid Res 2008:49:1466-76.

[54] Mori K, Matsuda H. Synthesis of sphingosine relatives. 10. Synthesis of (2S,3R,4E)-1$\mathrm{O}(\beta$-D-glucopyranosyl)-N-[30-linoleoyloxy)triacontanoyl]-4-icosasphingenine, a new esterified cerebroside isolated from human and pig epidermis. Liebigs Ann Chem 1991:529-35.
[55] Engelbrecht T, Hauß T, Süß K, Vogel A, Roark M, Feller SE, et al. Characterisation of a new ceramide EOS species: synthesis and investigation of the thermotropic phase behaviour and influence on the bilayer architecture of stratum corneum lipid model membranes. Soft Matter 2011;7:8998-9011.

[56] Woese CR, Fox GE. Phylogenetic structure of prokaryotic domains: the primary kingdom. Proc Natl Acad Sci U S A 1977;74:5088-90.

[57] Furmannn JF, McCallum K, Davis A. Novel major archaebacterial group from marine plankton. Nature 1992;356:148-9.

[58] Gabriel JL, Chong PLG. Molecular modeling of archaebipolar tetraether lipid membranes. Chem Phys Lipids 2000;105:193-200.

[59] Gliozzi A, Relini A, Chong PLG. Structure and permeabilities of biomimetic membranes of bolaform archaeal tetraether lipids. J Membr Sci 2002;206:131-47.

[60] Zhang D, Poulter CD. Biosynthesis of archaebacterial ether lipids - formation of ether linkages by prenyltransferases. J Am Chem Soc 1993;115:1270-7.

[61] Meister A, Blume A. Self-assembly of bipolar amphiphiles. Curr Opin Colloid Interface Sci 2007;12:138-47.

[62] Markowski T, Drescher S, Meister A, Hause G, Blume A, Dobner B. Synthesis of optical pure diglycerol tetraether model lipids with non-natural branching pattern. Eur J Org Chem 2011:5894-904.

[63] Blöcher D, Gutermann R, Henkel B, Ring K. Physicochemical characterization of tetraether lipids from Thermoplasma acidophilum. Differential scanning calorimetry studies on glycolipids and glycerophospholipids. Biochim Biophys Acta $1984 ; 778: 74-80$ 